\title{
Normalization of Collisional Decoherence: Squaring the Delta Function, and an Independent Cross-Check
}

\author{
Stephen L. Adler \\ Institute for Advanced Study \\ Princeton, NJ 08540
}

Send correspondence to:

Stephen L. Adler

Institute for Advanced Study

Einstein Drive, Princeton, NJ 08540

Phone 609-734-8051; FAX 609-924-8399; email adler@ias.edu 


\begin{abstract}
We show that when the Hornberger-Sipe calculation of collisional decoherence is carried out with the squared delta function a delta of energy instead of a delta of the absolute value of momentum, following a method introduced by Diósi, the corrected formula for the decoherence rate is simply obtained. The results of Hornberger and Sipe and of Diósi are shown to be in agreement. As an independent cross-check, we calculate the mean squared coordinate diffusion of a hard sphere implied by the corrected decoherence master equation, and show that it agrees precisely with the same quantity as calculated by a classical Brownian motion analysis.
\end{abstract}




\section{Introduction}

The calculation of collisional decoherence was initiated by Joos and Zeh [1], with generalizations of their result and corrections to the overall normalization given in papers of Gallis and Fleming [2], Dodd and Halliwell [3], and Hornberger and Sipe [4]. An even more general calculation of collisional decoherence was also given by Diósi [5], but this was not known to Hornberger and Sipe, while noted by Dodd and Halliwell, as well as in the master equation papers of Altenmüller, Müller, and Schenzle [6] and of Vacchini [7]. A difficulty encountered in refs [1], [2], and [4] is the appearance of a squared delta function of the absolute value of momentum in the calculation of the decoherence rate. To circumvent this, Hornberger and Sipe carried out a careful wave packet analysis, which gives an answer smaller by a factor of $2 \pi$ than that given by Gallis and Fleming, and this result is in agreement with experiment [8].

In the course of an alternative derivation of the corrected result, following the "traditional approach" of refs [1] and [2], Hornberger and Sipe introduce a rule in which the squared delta function of absolute value of momentum is evaluated in terms of an inverse scattering cross section, which drops out later in their calculation. This mixing of kinematic quantities (such as a squared delta function) and dynamical ones (such as a cross section) is unconventional, and Hornberger and Sipe describe this part of their calculation as speculative. In Sec. 2 we show that an entirely conventional completion of the Hornberger-Sipe calculation is possible, if one follows the method used in the earlier and more general master equation derivation given by Diósi, and also used in the derivation of Dodd and Halliwell. These authors retain the delta function of energy that appears as the $\mathcal{T}$-matrix coefficient, rather than converting this delta function to a delta function of the absolute value of mo- 
mentum. This makes a difference when squaring the delta function. For a squared delta function of energy, one can use the standard rule of evaluating $\delta(0)$ in terms of the elapsed conjugate time variable, as is done in the usual textbook "golden rule" calculation. For a delta function of absolute value of momentum, it is not so clear what to use as the corresponding conjugate variable when taking the square, and this appears to be the root of the difficulties in the earlier calculations of refs [1], [2], and [4].

In Sec. 3 we compare the Hornberger-Sipe and Diósi results and show that they are the same; hence Diósi's 1995 calculation appears to be the first giving the correct result for the collisional decoherence rate. In Sec. 4 we give an independent check of the corrected expression for collisional decoherence, by using the corresponding master equation to evaluate

the scattering-induced translational Brownian diffusion of a hard sphere in the geometric scattering limit. Planck's constant cancels out in this calculation, and so the result obtained this way can be directly compared with the classical Brownian diffusion of a hard sphere, and the two calculations are in precise agreement.

\section{Calculation using a squared energy delta function}

To keep this section concise, we will use the notation of Hornberger and Sipe, and give just a brief summary of their calculation up to the point where our treatment begins to differ from theirs. We consider a Brownian particle in a bath of $N$ scattering particles of mass $m$, contained in normalization volume $\Omega$. In the dilute case, the scatterings of the bath particles from each other can be neglected, and their scatterings from the Brownian particle are independent of one another. Then the effect of the $N$ bath particles is obtained by considering the effect of a single bath particle, and multiplying by $N$ at the end of the 
calculation. For a single scattering, the effect of the collision is to change the density matrix $\rho_{0}\left(\mathbf{R}_{1}, \mathbf{R}_{2}\right)$ to

$$
\rho\left(\mathbf{R}_{1}, \mathbf{R}_{2}\right)=\eta\left(\mathbf{R}_{1}, \mathbf{R}_{2}\right) \rho_{0}\left(\mathbf{R}_{1}, \mathbf{R}_{2}\right)
$$

with the factor $\eta\left(\mathbf{R}_{1}, \mathbf{R}_{2}\right)$ given by

$$
\eta\left(\mathbf{R}_{1}, \mathbf{R}_{2}\right)=\operatorname{tr}_{\text {bath }}\left\{e^{-i \mathbf{p} \cdot \mathbf{R}_{2} / \hbar} \mathcal{S}_{0}^{\dagger} e^{i \mathbf{p} \cdot\left(\mathbf{R}_{2}-\mathbf{R}_{1}\right) / \hbar} \mathcal{S}_{0} e^{i \mathbf{p} \cdot \mathbf{R}_{1} / \hbar} \rho^{\text {bath }}\right\}
$$

Here $\mathcal{S}_{0}$ is the scattering matrix, and $\rho^{\text {bath }}$ corresponds to an ensemble momentum space weighting

$$
\int d \mathbf{p} \mu(\mathbf{p})
$$

where for a thermal ensemble with $\beta=(k T)^{-1}$ one has

$$
\mu(\mathbf{p})=\left(\frac{\beta}{2 \pi m}\right)^{3 / 2} e^{-\beta \mathbf{p}^{2} /(2 m)}
$$

Substituting $\mathcal{S}_{0}=1+i \mathcal{T}_{0}$, evaluating the bath trace in a momentum basis, inserting a complete set of intermediate states, changing from box to continuum normalization, and using the unitarity relation $i\left(\mathcal{T}_{0}-\mathcal{T}_{0}^{\dagger}\right)=-\mathcal{T}_{0}^{\dagger} \mathcal{T}_{0}$, Hornberger and Sipe show that Eq. (2) takes the form

$$
\eta\left(\mathbf{R}_{1}, \mathbf{R}_{2}\right)=\int d \mathbf{p} \mu(\mathbf{p})\left[1-\frac{(2 \pi \hbar)^{3}}{\Omega} \int d \mathbf{p}^{\prime}\left(1-e^{i\left(\mathbf{p}-\mathbf{p}^{\prime}\right) \cdot\left(\mathbf{R}_{1}-\mathbf{R}_{2}\right) / \hbar}\right)\left|\left\langle\mathbf{p}^{\prime}\left|\mathcal{T}_{0}\right| \mathbf{p}\right\rangle\right|^{2}\right]
$$

This equation, which is Eq. (51) of Hornberger and Sipe, and without the weighting over $\mu(\mathbf{p})$ also corresponds to the first line of Eq. (2.11) of Gallis and Fleming, will be the starting point for our analysis.

Denoting the elapsed time in the scattering process by $T$, so that Eqs. (1) and (4a) implicitly refer to time $T$, and recalling that $1=\int d \mathbf{p} \mu(\mathbf{p})$, we can rewrite Eqs. (1) and (4a) 
as

$$
\begin{aligned}
& \rho\left(\mathbf{R}_{1}, \mathbf{R}_{2} ; T\right)-\rho\left(\mathbf{R}_{1}, \mathbf{R}_{2} ; 0\right)=\left(\eta\left(\mathbf{R}_{1}, \mathbf{R}_{2} ; T\right)-1\right) \rho_{0}\left(\mathbf{R}_{1}, \mathbf{R}_{2}\right) \\
= & -\rho_{0}\left(\mathbf{R}_{1}, \mathbf{R}_{2}\right) \int d \mathbf{p} \mu(\mathbf{p}) \frac{(2 \pi \hbar)^{3}}{\Omega} \int d \mathbf{p}^{\prime}\left(1-e^{i\left(\mathbf{p}-\mathbf{p}^{\prime}\right) \cdot\left(\mathbf{R}_{1}-\mathbf{R}_{2}\right) / \hbar}\right)\left|\left\langle\mathbf{p}^{\prime}\left|\mathcal{T}_{0}\right| \mathbf{p}\right\rangle\right|^{2}
\end{aligned}
$$

Our next task will be to evaluate the squared matrix element appearing in the integrand of Eq. (4b).

The general $\mathcal{T}_{0}$ matrix element $\left\langle\mathbf{q}_{2}\left|\mathcal{T}_{0}\right| \mathbf{q}_{1}\right\rangle$ can as usual be expressed in terms of the scattering amplitude $f\left(\mathbf{q}_{2}, \mathbf{q}_{1}\right)$ and an energy-conserving delta function,

$$
\begin{aligned}
\left\langle\mathbf{q}_{2}\left|\mathcal{T}_{0}\right| \mathbf{q}_{1}\right\rangle & =\frac{1}{2 \pi \hbar m} \delta\left(E_{2}-E_{1}\right) f\left(\mathbf{q}_{2}, \mathbf{q}_{1}\right) \\
& =\frac{1}{2 \pi \hbar q_{2}} \delta\left(q_{2}-q_{1}\right) f\left(\mathbf{q}_{2}, \mathbf{q}_{1}\right) .
\end{aligned}
$$

Instead of using the second line of Eq. (5) to form the square of the $\mathcal{T}_{0}$ matrix element, we will use the first line, using the second line only after the delta function of zero energy argument has been evaluated. Thus, we have

$$
\left|\left\langle\mathbf{q}_{2}\left|\mathcal{T}_{0}\right| \mathbf{q}_{1}\right\rangle\right|^{2}=\frac{1}{(2 \pi \hbar m)^{2}} \delta^{2}\left(E_{2}-E_{1}\right)\left|f\left(\mathbf{q}_{2}, \mathbf{q}_{1}\right)\right|^{2}
$$

Using the Fourier representation for the energy delta function,

$$
\delta\left(E_{2}-E_{1}\right)=\frac{1}{2 \pi \hbar} \int_{-\infty}^{\infty} d t \exp \left[i\left(E_{2}-E_{1}\right) t / \hbar\right]
$$

we find

$$
\begin{aligned}
\delta^{2}\left(E_{2}-E_{1}\right) & =\delta\left(E_{2}-E_{1}\right) \delta(0) \\
& =\delta\left(E_{2}-E_{1}\right) \frac{1}{2 \pi \hbar} \int d t \\
& =\delta\left(E_{2}-E_{1}\right) \frac{T}{2 \pi \hbar} \\
& =\frac{m}{q_{2}} \frac{T}{2 \pi \hbar} \delta\left(q_{2}-q_{1}\right)
\end{aligned}
$$

where in the final line we have converted the energy delta function to a delta function of the absolute value of the three-momentum. Here $T$ is the elapsed time interval, which we 
assume to be longer than the time for a single scattering, but still short compared to the characteristic decoherence time of the Brownian particle in the $N$-particle bath.

Replacing $\mathbf{q}_{2}, \mathbf{q}_{1}$ by $\mathbf{p}^{\prime}, \mathbf{p}$ respectively, substituting Eqs. (7b) and (5) into Eq. (4b), and writing $d \mathbf{p}^{\prime}=d \hat{n}\left(p^{\prime}\right)^{2} d p^{\prime}$, with $d \hat{n}$ a solid angle differential, we can immediately integrate out the delta function of the absolute value of momentum. Using

$$
\frac{(2 \pi \hbar)^{3}}{\Omega} \frac{1}{(2 \pi \hbar m)^{2}} p^{2} \frac{m}{p} \frac{T}{2 \pi \hbar}=\frac{T}{\Omega} \frac{p}{m}
$$

we thus get

$$
\begin{aligned}
\rho\left(\mathbf{R}_{1}, \mathbf{R}_{2} ; T\right)-\rho\left(\mathbf{R}_{1}, \mathbf{R}_{2} ; 0\right)= & -\rho_{0}\left(\mathbf{R}_{1}, \mathbf{R}_{2}\right) \\
& \times \frac{T}{\Omega} \int d \mathbf{p} \mu(\mathbf{p}) \frac{p}{m} \int d \hat{n}\left(1-e^{i(\mathbf{p}-p \hat{n}) \cdot\left(\mathbf{R}_{1}-\mathbf{R}_{2}\right) / \hbar}\right)|f(p \hat{n}, \mathbf{p})|^{2}
\end{aligned}
$$

Multiplying by $N$ to take account of the fact that each of the $N$ bath particles makes a contribution equal to Eq. (8b), denoting the bath density $N / \Omega$ by $n$, dividing by $T$, and finally passing to the limit of small $T$, we get the result

$$
\frac{\partial \rho\left(\mathbf{R}_{1}, \mathbf{R}_{2} ; t\right)}{\partial t}=-F\left(\mathbf{R}_{1}-\mathbf{R}_{2}\right) \rho\left(\mathbf{R}_{1}, \mathbf{R}_{2} ; t\right)
$$

with

$$
F\left(\mathbf{R}_{1}-\mathbf{R}_{2}\right)=n \int d \mathbf{p} \mu(\mathbf{p}) \frac{p}{m} \int d \hat{n}\left(1-e^{i(\mathbf{p}-p \hat{n}) \cdot\left(\mathbf{R}_{1}-\mathbf{R}_{2}\right) / \hbar}\right)|f(p \hat{n}, \mathbf{p})|^{2}
$$

This is the form of the final result for the decoherence-induced master equation given in Eq. (55), and in the unnumbered immediately preceding equation, of Hornberger and Sipe [4]. By defining $\nu(p)$ by

$$
\mu(\mathbf{p}) d \mathbf{p}=\frac{\nu(p) d p d \hat{s}}{4 \pi}
$$


with $d \hat{s}$ a second solid angle differential, so that $\int_{0}^{\infty} d p \nu(p)=1$, Hornberger and Sipe also rewrite Eq. (9b) in the equivalent form (after a relabeling of the integration variables)

$$
F(\mathbf{R})=n \int_{0}^{\infty} d q \nu(q) \frac{q}{m} \int \frac{d \hat{n}_{1} d \hat{n}_{2}}{4 \pi}\left(1-e^{i q\left(\hat{n}_{1}-\hat{n}_{2}\right) \cdot \mathbf{R} / \hbar}\right)\left|f\left(q \hat{n}_{2}, q \hat{n}_{1}\right)\right|^{2}
$$

In the limit of large $\mathbf{R}$, the exponential term in Eqs. (9b) and (10b) averages to zero provided that $\hat{n}_{2} \neq \hat{n}_{1}$, while in the forward direction $\hat{n}_{2}=\hat{n}_{1}$ the integrand in Eqs. (9b) and (10b) vanishes for all $\mathbf{R}$. Hence one has

$$
\begin{aligned}
F(\mathbf{R} \rightarrow \infty) & =n \int_{0}^{\infty} d q \nu(q) \frac{q}{m} \int_{\hat{n}_{2} \neq \hat{n}_{1}} \frac{d \hat{n}_{1} d \hat{n}_{2}}{4 \pi}\left|f\left(q \hat{n}_{2}, q \hat{n}_{1}\right)\right|^{2} \\
& =n \int_{0}^{\infty} d q \nu(q) \frac{q}{m} \sigma(q),
\end{aligned}
$$

with $\sigma(q)$ the total cross section (excluding a possible delta function contribution to the forward diffraction peak). In other words, the large $\mathbf{R}$ asymptote of $F(\mathbf{R})$ is the thermal ensemble average $\langle n v \sigma\rangle_{\mathrm{AV}}$ of the non-forward scattering rate $n(q / m) \sigma(q)$. Correspondingly, from Eqs. (4b) and (8b), as modified by multiplication by the factor $N$, we see that $\eta\left(\mathbf{R}_{1}, \mathbf{R}_{2} ; T\right)$ $=1-T F\left(\mathbf{R}_{1}-\mathbf{R}_{2}\right)$ has the large $\mathbf{R}$ asymptote $\eta(\infty ; T)=1-T\langle n v \sigma\rangle_{\mathrm{AV}}$. Thus $\eta(\infty ; T)$ vanishes for $T$ equal to the inverse of the averaged non-forward scattering rate, a result reminiscent of, but not identical to, the condition $\eta(\infty)=0$ imposed by Hornberger and Sipe on the single collision decoherence function in their Eq. (45), on which they base their method for evaluating the square of a delta function of the absolute value of momentum. (We emphasize, however, that in the calculation leading to Eq. (9b) we have not fixed $T$ by imposing such a condition.)

\section{Comparison of the Hornberger-Sipe and Diósi Results}

The calculation of Diósi includes effects of recoil of the Brownian particle; we show in this section that in the limit of an infinitely heavy Brownian particle, the results of 
Hornberger-Sipe and of Diósi are in agreement. When recoil is neglected, Diósi's result is his Eq. (19), which reads

$$
\frac{d \rho}{d t}=n_{0} \int d E d \Omega_{i} d \Omega_{f} k^{2} \frac{d \sigma(\theta, E)}{d \Omega_{f}} \rho^{\mathcal{E}}\left(\mathbf{k}_{i}\right)\left(V_{\mathbf{k}_{f} \mathbf{k}_{i}} \rho V_{\mathbf{k}_{f} \mathbf{k}_{i}}^{\dagger}-\frac{1}{2}\left\{V_{\mathbf{k}_{f} \mathbf{k}_{i}}^{\dagger} V_{\mathbf{k}_{f} \mathbf{k}_{i}}, \rho\right\}\right) .
$$

Substituting into Eq. (11a) Diósi's Eq. (20) (with the Brownian particle recoil term dropped),

$$
V_{\mathbf{k}_{f} \mathbf{k}_{i}}=\exp \left(-i \mathbf{k}_{f i} \cdot \mathbf{q}\right)=\exp \left(-\mathbf{i}\left(\mathbf{k}_{\mathbf{f}}-\mathbf{k}_{\mathbf{i}}\right) \cdot \mathbf{q}\right)
$$

taking the matrix element of Eq. (11a) between $\mathbf{q}$ eigenstates $\left\langle\mathbf{R}_{1}\right|$ and $\left|\mathbf{R}_{2}\right\rangle$, and writing

$$
\rho\left(\mathbf{R}_{1}, \mathbf{R}_{2} ; t\right)=\left\langle\mathbf{R}_{1}|\rho(t)| \mathbf{R}_{2}\right\rangle
$$

we get

$$
\frac{d \rho\left(\mathbf{R}_{1}, \mathbf{R}_{2} ; t\right)}{d t}=-n_{0} \int d E d \Omega_{i} d \Omega_{f} k^{2} \frac{d \sigma(\theta, E)}{d \Omega_{f}} \rho^{\mathcal{E}}\left(\mathbf{k}_{i}\right)\left(1-e^{i\left(\mathbf{k}_{i}-\mathbf{k}_{f}\right) \cdot\left(\mathbf{R}_{1}-\mathbf{R}_{2}\right)}\right) \rho\left(\mathbf{R}_{1}, \mathbf{R}_{2} ; t\right)
$$

Taking account of the fact that $d E=d k^{2} /(2 m)=(k / m) d k$, together with $d \sigma(\theta, E) / d \Omega_{f}=$ $|f|^{2}$, where $f$ is the scattering amplitude, along with $\rho^{\mathcal{E}}\left(\mathbf{k}_{i}\right)=\mu\left(\mathbf{k}_{i}\right)$ and some obvious relabelings of variables, one sees that Eq. (12) is identical to Eqs. (9a) and (9b) that follow from the analysis of Hornberger and Sipe.

Diósi also gives the expansion of Eq. (12) to leading order in $\mathbf{R}=\mathbf{R}_{1}-\mathbf{R}_{2}$. Working now in the other direction, from Eq. (10b), the simplest way to find the leading order $\mathbf{R}$ dependence is to note that $F(\mathbf{R})$ is a rotationally invariant function of $\mathbf{R}$. Hence it suffices to evaluate the average over the direction of $\mathbf{R}$. Expanding out the exponential in Eq. (10b), we have

$$
1-e^{i q\left(\hat{n}_{1}-\hat{n}_{2}\right) \cdot \mathbf{R} / \hbar}=1-i q\left(\hat{n}_{1}-\hat{n}_{2}\right) \cdot \mathbf{R} / \hbar+\frac{1}{2}\left(q^{2} / \hbar^{2}\right)\left[\left(\hat{n}_{1}-\hat{n}_{2}\right) \cdot \mathbf{R}\right]^{2}+\ldots
$$


The average of $\left[\left(\hat{n}_{1}-\hat{n}_{2}\right) \cdot \mathbf{R}\right]^{2}$ over the direction of $\mathbf{R}$ is

$$
\begin{aligned}
\frac{1}{3} R^{2} \sum_{i}\left[\left(\hat{n}_{1}-\hat{n}_{2}\right) \cdot \hat{i}\right]^{2} & =\frac{1}{3} R^{2}\left(\hat{n}_{1}-\hat{n}_{2}\right)^{2} \\
& =\frac{2}{3} R^{2}\left(1-\hat{n}_{1} \cdot \hat{n}_{2}\right)=\frac{4}{3} R^{2} \sin ^{2}(\theta / 2),
\end{aligned}
$$

with $\theta$ the angle (the scattering angle) between $\hat{n}_{1}$ and $\hat{n}_{2}$. Substituting Eqs. $(13 \mathrm{a}, \mathrm{b})$ into Eq. (10b), we get

$$
F(\mathbf{R})=R^{2} \Lambda
$$

with

$$
\begin{aligned}
\Lambda & =\frac{2}{3} \frac{n}{\hbar^{2}} \int_{0}^{\infty} d q \nu(q) \frac{q}{m} q^{2} \int \frac{d \hat{n}_{1} d \hat{n}_{2}}{4 \pi} \sin ^{2}(\theta / 2)\left|f\left(q \hat{n}_{2}, q \hat{n}_{1}\right)\right|^{2} \\
& =\frac{2}{3} \frac{n}{\hbar^{2}} \int d \mathbf{q} \mu(\mathbf{q}) \frac{q}{m} q^{2} \int d \hat{n}_{2} \sin ^{2}(\theta / 2)\left|f\left(q \hat{n}_{2}, q \hat{n}_{1}\right)\right|^{2}
\end{aligned}
$$

With the shifts in notation noted above, this equation for $\Lambda$ is identical to Eq. (22) of Diósi, which gives what he terms the diffusion parameter $D_{p p}$.

\section{Comparison of decoherence-based and classical calculations of Brownian translational diffusion}

Let us now apply the result of Eqs. $(14 a, b)$ to the quantum Brownian motion of a Brownian particle of mass $M$ and radius $a$, with $a$ large enough so that $p a>>1$ for important bath particle momenta. In this case the quantum scattering differential cross section consists of two parts [9]: an isotropic part, with an integrated cross section contribution of $\pi a^{2}$, and a forward diffraction peak, again contributing cross section $\pi a^{2}$. Since the integrand of Eq. (14b) vanishes for forward scattering, the forward diffraction peak makes no contribution. Thus we can evaluate the integrals by taking the scattering amplitude to be a constant, $f\left(q \hat{n}_{2}, q \hat{n}_{1}\right)=F$, with

$$
\int d \hat{s}|F|^{2}=4 \pi|F|^{2}=\pi a^{2}
$$


that is, with

$$
|F|^{2}=a^{2} / 4
$$

Substituting into Eq. (14b), we then find that

$$
\Lambda=\frac{n \pi a^{2}\left\langle q^{2} v\right\rangle_{\mathrm{AV}}}{3 \hbar^{2}}
$$

Here we have defined

$$
\left\langle q^{2} v\right\rangle_{\mathrm{AV}}=\int_{0}^{\infty} d q \nu(q) q^{2} \frac{q}{m}=4(m / \pi)^{\frac{1}{2}}(2 k T)^{\frac{3}{2}}
$$

where we used the expression

$$
\nu(q)=4 \pi q^{2}\left(\frac{\beta}{2 \pi m}\right)^{\frac{3}{2}} e^{-\beta q^{2} /(2 m)}
$$

which follows from the definitions of Eqs. (3b) and (10a), to evaluate the thermal average.

Substituting Eq. (14a) into Eq. (9a), we then get

$$
\frac{\partial \rho\left(\mathbf{R}_{1}, \mathbf{R}_{2} ; t\right)}{\partial t}=-\Lambda\left(\mathbf{R}_{1}-\mathbf{R}_{2}\right)^{2} \rho\left(\mathbf{R}_{1}, \mathbf{R}_{2} ; t\right)
$$

which with Eq. (11c) is equivalent to the operator equation

$$
\frac{\partial \rho(t)}{\partial t}=-\Lambda \sum_{j=1}^{3}\left[R_{j},\left[R_{j}, \rho(t)\right]\right]
$$

with $R_{j}$ denoting the Cartesian components of $\mathbf{R}$. Adding the kinetic energy term to the differential equation for $\rho(t)$, we get the total evolution equation

$$
\frac{\partial \rho(t)}{\partial t}=-\frac{i}{\hbar}\left[H_{\mathrm{kin}}, \rho(t)\right]-\Lambda \sum_{j=1}^{3}\left[R_{j},\left[R_{j}, \rho(t)\right]\right]
$$

with the kinetic Hamiltonian for the Brownian particle given by

$$
H_{\mathrm{kin}}=\sum_{j=1}^{3} \frac{P_{j}^{2}}{2 M}
$$


Here $P_{j}$ is the momentum operator corresponding to the coordinate operator $R_{j}$, so that $\left[R_{j}, P_{k}\right]=i \hbar \delta_{j k}$

From Eqs. (19a) and (19b), one can calculate the mean squared coordinate diffusion as a function of time, for a Brownian particle that starts at $\mathbf{R}=\mathbf{0}$ at $t=0$ with zero drift velocity. This calculation in the one-dimensional case is given in Adler [10], by constructing a generating function for the trace of $\rho(t)$ multiplied by an arbitrary polynomial constructed from $R_{j}$ and $P_{k}$. For the mean square coordinate deviation, the result on converting to the present notation is (with no sum implied over $j$ )

$$
\left\langle R_{j}^{2}\right\rangle=\operatorname{tr} \rho(t) R_{j}^{2}=\frac{2 \Lambda \hbar^{2} t^{3}}{3 M^{2}}
$$

Substituting Eq. (16) for $\Lambda$ and Eq. (17a) for the thermal average of $q^{2} v$, we get finally (again with $j$ unsummed)

$$
\left\langle R_{j}^{2}\right\rangle=C(k T)^{\frac{3}{2}} n m^{\frac{1}{2}} a^{2} t^{3} / M^{2}
$$

with

$$
C=\frac{16}{9}(2 \pi)^{\frac{1}{2}}
$$

Note that the Planck constant $\hbar$ has dropped out of this result. Hence the formula of Eqs. (20b,c) is a classical result, and should be recoverable by a purely classical calculation.

The formulas needed for a classical Brownian motion evaluation of $\left\langle R_{j}^{2}\right\rangle$ are summarized in a recent paper by Collett and Pearle [11]. Their Eq. (2.2) gives (again with $j$ unsummed)

$$
\left\langle R_{j}^{2}\right\rangle=\frac{2 k T \xi t^{3}}{3 M^{2}}
$$

with $\xi$ a viscosity factor, which for a sphere of radius $a$ in a dilute bath is given by their 
Eq. (2.5),

$$
\xi=\frac{8}{3} n a^{2}(2 \pi m k T)^{\frac{1}{2}} .
$$

Substituting Eq. (21b) into Eq. (21a) then gives a result identical to Eqs. (20b,c) above. Since the result of Eqs $(20 \mathrm{~b}, \mathrm{c})$ is directly proportional to the normalization constant in the collisional decoherence rate, this agreement gives added confirmation of the correctness of $\mathrm{Eq}(9 \mathrm{~b})$.

\section{Acknowledgments and Addenda}

This work was supported in part by the Department of Energy under Grant \#DEFG02-90ER40542, and was done while the author was at the Aspen Center for Physics. I wish to Philip Pearle for alerting me to reference [9], which was the initial stimulus for this investigation, and Jonathan Halliwell for bringing references [2] - [4] to my attention. A related discussion of the derivation of the decoherence master equation is given in a recent article of Halliwell [12]. I also wish to thank Klaus Hornberger for an informative email correspondence, and for noting a recent paper [13], in which he extends the method of ref [4] to the case of a finite mass Brownian particle. Finally, Alexander Pechen has pointed out a recent paper [14], giving further references, pertaining to the microscopic derivation of the master equation for a quantum system interacting with a dilute environment. 


\section{References}

[1] Joos E and Zeh H D (1985) Z. Phys. B: Condens. Matt. 59223.

[2] Gallis M R and Fleming G N (1990) Phys. Rev. A 4238.

[3] Dodd P J and Halliwell J J (2003) Phys. Rev. D 67 105018. See also the related recent article of Halliwell [10].

[4] Hornberger K and Sipe J E (2003) Phys. Rev. A 68 012105. See also the related recent article of Hornberger [11].

[5] Diósi L (1995) Europhys. Lett. 3063 (1995).

[6] Altenmüller T P, Müller R and Schenzle A (1997) Phys. Rev. A 562959.

[7] Vacchini B (2000) Phys. Rev. Lett. 841374.

[8] Hornberger K, Uttenthaler S, Brezger B, Hackermüller L, Arndt M and Zeilinger A (2003) Phys. Rev. Lett. 90160401. 
[9] Schiff L I (1968) Quantum Mechanics, 3rd ed (New York: McGraw-Hill) pp 124-126.

[10] Adler S L (2005) J. Phys. A: Math. Gen. 382729.

[11] Collett B and Pearle P (2003) Found. Phys. 331495.

[12] Halliwell J J (2006) "Two Derivations of the Master Equation of Quantum Brownian Motion" to appear in J. Phys. A: Math. Gen..

[13] Hornberger K (2006) "Master equation for a quantum particle in a gas" arXiv:quantph/0607085.

[14] Pechen A (2006) "White noise approach to the low density limit of a quantum particle in a gas" arXiv:quant-ph/0607134. 